and the software developed for segmentation and optical density analyses.

Methods The non-dominant hand is radiographed together with an aluminium wedge used for calibration and correction of the variability associated with the $\mathrm{XR}$ technique and digitalisation process. A single exposure at $46 \mathrm{kV}$ is performed and the resulting film is read with an AgfaScan T1200 device with predefined settings.

Results To assess the reproducibility of our technique, XR hand films of 17 patients were scanned 5 times in different days. CV was found to be 0,913 (95\% CI $0,650-1,825)$ for the middle phalanx; 0,632 (95\% CI $0,470-1,265)$ for the proximal phalanx; and 1,194 (95\% CI 0,918-2,387) for the central area of the third metacarpal. A test-retest experiment with 2 films in 50 patients gave an almost perfect internal correlation (Pearson's $\mathrm{R}$ of 0,$985 ; 0,995$ and 0,986 ) for these same sites.

We also validated our technique in a sample of 171 women referred by general practitioners with a clinical diagnosis of osteoporosis. Construct validation was performed against conventional lumbar spine and hip DXA (Lunar DPX). Also,78 of these women had a phalanx DXA measurement with a commercial device (AccuDXA).

Correlations between BMD in several sites are described below:

\begin{tabular}{lllllll}
\multicolumn{6}{l}{ Abstract SAT0203 } & Table 1 \\
\hline & Prox Phal & MCP & Spine & Hip & AccuDXA & Age \\
\hline Middle Phal & $0,851^{*}$ & $0,755^{*}$ & $0,562^{*}$ & $0,565^{*}$ & $0,674^{*}$ & $-0,264$ \\
Prox Phal & & $0,845^{*}$ & $0,551^{*}$ & $0,580^{*}$ & $0,742^{*}$ & $-0,366^{*}$ \\
MCP & & & $0,548^{*}$ & $0,603^{*}$ & $0,713^{*}$ & $-0,430^{*}$ \\
Spine & & & & $0,708^{*}$ & $0,510^{*}$ & $-0,385$ \\
Hip & & & & $0,623^{*}$ & $-0,495^{*}$ \\
AccuDXA & & & & & & $-0,394^{*}$ \\
\hline Correlation among different BMD mesaurement procedures.
\end{tabular}

Conclusion The reproducibility and internal correlation of measurements made with our newly developed technique is very high for the three areas of the hand studied.

Correlation of BMD of the hand with BMD in the lumbar spine and hip is moderate and similar to that found among these places themselves.

\section{SAT0204 INVESTIGATION OF FLUOROQUINOLONE-INDUCED MYALGIA USING 31P MAGNETIC RESONANCE SPECTROSCOPY AND IN VITRO CONTRACTURE TESTS}

S Guis, D Bendahan, G Kozak-Ribbens, JP Mattei, Y Le Fur, D Figarella-Branger, J Jouglard, PJ Cozzone. CRMBM, UMR CNRS N6612, Faculté de Médecine de La Timone, Marseille, France

10.1136/annrheumdis-2001.701

Background Adverse rheumatological effects of fluoroquinolone treatment such as tendinopathies, arthralgia and myalgia have been reported. The exact mechanism of fluoroquinolone toxicity remains unclear.

Objectives To investigate muscle function in patients suffering from severe myalgia resulting from fluoroquinolone treatment. We used histology, in vitro contracture tests (IVCT) and 31P magnetic resonance spectroscopy (31P MRS) to explore muscular contraction and metabolism.
Methods Three patients were investigated suffering after fluoroquinolone treatment from myalgia, hyperalgia tendinopathy and arthralgia. Control group included nine subjects, all of them were free of any muscle disease.

Muscle biopsies were performed on the left biceps, IVCT were performed in accordance with the protocol recommended by European Malignant Hyperpyrexia Group (EMHG). 31P MRS concicted to MR spectra of forearm flexor muscles were recorded at 4.7 $\mathrm{T}$ throughout a rest-exercise-recovery protocol.

Results No specific histologic anomalies were observed in muscle biopsies. In vitro contracture tests diagnosed one patient as susceptible to Malignant hyperthermia. 31P MRS detected in all patients a significant reduction of $\mathrm{pH}$ magnitude changes measured at end of exercise and a faster rate of proton efflux measured during recovery.

Conclusion Muscle abnormalities described in the present study of three patients after fluoroquinolone treatment clearly pointed out the organicity of fluoroquinolone-induced myalgia. All three patients displayed an aceleration of proton efflux and a limited acidosis likely in relation with changes of transporters involving in the transport of $\mathrm{Na}+, \mathrm{H}+$ and $\mathrm{Ca}^{2+}$.

\section{SAT0205 OSTEOPOROSIS: A NEW METHOD OF SCREENING}

N Navarro, M Castellanos, MP Lisbona, C Galisteo, MC Garcia, MC Sanmartí, C Alegre. Rheumatology, Hospital Malalties Reumatiques, Barcelona, Spain

\subsection{6/annrheumdis-2001.702}

\section{Background}

Objectives Usefulness of calcaneus densitometry as a method of screening in osteoporosis.

Methods Study of 69 women with osteoporosis, with DMO (L2L4, Ward) and a comparison of the results obtained with a calcaneus densitometry (photonic dual measure, Lunar PIXI model). We considered osteoporosis when we find $-2.5 \mathrm{SD}$ in DMO and $-1.6 \mathrm{SD}$ in the calcaneus densitometry.

Results After comparing the DMO lumbar results and DMO Ward, individually, with results of calcaneus densitometry, we observed a coefficient correlation of 0.5 and 0.6 respectively.

Conclusion In our study the measures of the calcaneus densitometry have a relation to DMO, then we could consider it as a method of screening with patients we suspect of a osteoporosis.

\section{SAT0206 DYNAMIC MAGNETIC RESONANCE IMAGING OF THE WRIST: A COMPARISON OF PATIENTS WITH ACTIVE RHEUMATOID ARTHRITIS, RHEUMATOID ARTHRITIS IN REMISSION, AND PSORIATIC ARTHRITIS}

${ }^{1} \mathrm{MA}$ Cimmino, ${ }^{2} \mathrm{~A}$ lozzelli, ${ }^{2} \mathrm{~F}$ Magnaguagno, ${ }^{1} \mathrm{M}$ Parodi, ${ }^{2} \mathrm{C}$ Gandolfo, ${ }^{1} \mathrm{~T}$ Maio, ${ }^{2} \mathrm{E}$ Silvestri ${ }^{3} \mathrm{M}$ Palazzolo, ${ }^{3} \mathrm{~S}$ Innocenti, ${ }^{2} \mathrm{G}$ Garlaschi. ${ }^{1}$ Clinica Reumatologica, Dipartimento Di Medicina Interna, Università Di Genova, Genova, Italy; ${ }^{2}$ Dipartimento Di Medicina Sperimentale, Università Di Genova, Genova, Italy; ${ }^{3} R \& D$, Esaote, Genova, Italy

10.1136/annrheumdis-2001.703

Background Objective quantification of synovial membrane inflammation is an important goal of rheumatology research. Recently, magnetic resonance imaging (MRI) has been used to evaluate synovitis in rheumatoid arthritis (RA). ${ }^{1,2}$

Objectives Our work is concerned with an evaluation of synovitis in patients with active RA, RA in remission, and psoriatic arthritis (PsA) in comparison with normal controls (CO). 